\title{
Transient phosphorus diffusion from silicon and argon implantation damage
}

\author{
Martin D. Giles \\ University of Michigan, Department of Electrical Engineering and Computer Science, \\ Ann Arbor, Michigan 48109-2122
}

(Received 17 August 1992; accepted for publication 24 January 1993)

\begin{abstract}
We have investigated transient enhanced diffusion of phosphorus in silicon following implantation with silicon or argon ions at low doses. Both conditions show uphill diffusion of phosphorus due to the defect gradients, but the resulting profiles are quite different because of differences in the initial defect distributions. These experiments support an interstitial pair diffusion mechanism for phosphorus and show the importance of bulk recombination in determining defect distributions for argon damage annealing.
\end{abstract}

Ion-implanted dopants such as phosphorus have been observed to exhibit a transient enhancement in diffusivity by factors of 1000 or more during the initial stages of annealing. ${ }^{1-3}$ These large enhancements are due to large supersaturations of point defects caused by the implantation process which then influence dopant diffusion during annealing. In general, the annealing of implantation damage is a complex process involving the formation of extended defects of various types depending on the implantation species, energy, dose, and wafer temperature during implantation. Extended defects act as strong sinks for free point defects and lead to a strong spatial variation in transient enhancement of diffusivity. ${ }^{4,5}$ The problem is considerably simplified when the implantation dose is chosen below a threshold value such that no extended defects form during the annealing process. Studies of this regime have helped to improve our understanding of the underlying diffusion mechanisms and point defect properties.,

Implantation damage can be considered to consist of the generation of Frenkel pairs, each a silicon atom displaced from its lattice site to become an interstitial or interstitialcy and a vacancy left behind. Some of these pairs may recombine during the implantation process, while others remain until a high temperature annealing step is performed. For a silicon implant, there are additional silicon interstitial atoms added to the lattice corresponding to the implanted dose. Similarly, for a dopant implant, the same number of silicon interstitials are generated as the dopant is activated. At the low doses under consideration here, more than $90 \%$ of the implanted dopant is moved onto substitutional lattice sites and made electrically active by a 30 min anneal at $800^{\circ} \mathrm{C} .^{7}$ Almost all studies of transient enhanced diffusion have involved the implantation of dopant or silicon atoms, and so have had the same "pair +1 " point defect distribution controlling the transient enhanced diffusion effect. An alternative "pairs only" initial point defect distribution can be created if the implanted species does not tend to occupy substitutional lattice sites upon annealing and the dose is sufficiently low that extended defects are not generated in the lattice. This letter compares the transient enhanced diffusivity of phosphorus observed under these two conditions.

Earlier experiments corresponding to the pairs only defect generation case have been performed using high temperature proton irradiation (e.g., Refs. 8 and 9). NonFickian uphill diffusion effects have been observed, although there have been conflicting reports of whether dopant tends to diffuse toward or away from the projected range of the protons. ${ }^{10,11}$ Pitfalls in the analysis of these kinds of experiments have been discussed by $\mathrm{Hu} .{ }^{12}$ For the experiments described here, the goal was to create similar initial defect pair distributions so that the pairs only and the pairs +1 cases could be directly compared. Argon implantation was used to generate pairs only damage since it is the noble gas closest in mass to the silicon used for generating the pairs +1 damage. Although high dose argon implantation leads to the formation of a dislocation network and interstitial injection, ${ }^{13}$ the implantation doses used here were well below the threshold for amorphization of the lattice or extended defect formation upon annealing.

Our first experiment used $\langle 100\rangle 7 \mu \mathrm{m} p$-on- $p^{+}$epitaxial silicon wafers. First, a $125 \AA$ screen oxide was grown by dry oxidation at $900^{\circ} \mathrm{C}$. The wafers were then implanted with phosphorus at $150 \mathrm{keV}$ to a dose of $7 \times 10^{13} \mathrm{~cm}^{-2}$. All wafers were annealed in nitrogen at $800^{\circ} \mathrm{C}$ for $75 \mathrm{~min}$ to complete the initial transient diffusion of the implanted phosphorus. ${ }^{3}$ A second implantation step was performed using silicon or argon at $150 \mathrm{keV}$ to the same dose of $7 \times 10^{13} \mathrm{~cm}^{-2}$. Both samples were then annealed in nitrogen at $800^{\circ} \mathrm{C}$ for $30 \mathrm{~min}$. The phosphorus atom distribution was analyzed by secondary ion mass spectrometry (SIMS) using a CAMECA IMS- $3 f$ system in high mass resolution mode and a cesium sputtering beam.

The implanted phosphorus showed the expected transient diffusion enhancement during the first annealing step. Subsequently, implanting silicon and annealing causes a second transient diffusion enhancement. Figure 1 shows the phosphorus profile before and after the second annealing step. The same model as was used for the original phosphorus implant ${ }^{3}$ predicts the observed final phosphorus profile. This indicates that the point defect distribution created by implantation of phosphorus or silicon for the same dose and energy are very similar, as expected from the discussion above. Figure 2 shows the case where the second implantation used argon ions instead of silicon. The phosphorus diffusion during the second anneal is dramatically different from the first case, showing strong uphill diffusion to a region around $0.15 \mu \mathrm{m}$ from a region 


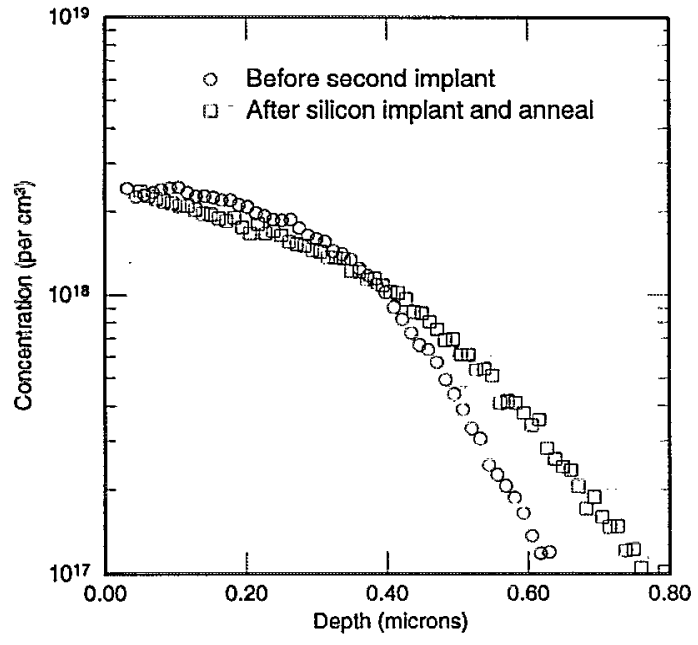

FIG. 1. Transient enhanced diffusion of implanted and annealed phosphorus following a second implantation with silicon at $150 \mathrm{keV}$ and dose $7 \times 10^{13} \mathrm{~cm}^{-2}$ and annealing at $800^{\circ} \mathrm{C}$ for $30 \mathrm{~min}$.

around $0.3 \mu \mathrm{m}$ and reduced diffusion in the tail of the profile compared to Fig. 1. Time evolution of the dopant distribution was not measured in these experiments, but it is expected to be similar to that found in previous work. ${ }^{3}$

In this first experiment, gradients of both phosphorus and point defects are present which may drive changes in the phosphorus profile. To consider a simpler case, a second experiment was performed starting with $\langle 100\rangle$ Czochralski wafers uniformly and heavily doped with phosphorus during crystal growth at a concentration of $7 \times 10^{18}$ $\mathrm{cm}^{-3}$. A screen oxide was grown at $900^{\circ} \mathrm{C}$, as before, and some wafers were implanted with silicon or argon at 150 $\mathrm{keV}$ to a dose of $10^{14} \mathrm{~cm}^{-2}$. All wafers were annealed in nitrogen at $800^{\circ} \mathrm{C}$ for $30 \mathrm{~min}$ and analyzed using SIMS. The resulting phosphorus profiles are shown in Fig. 3. The unimplanted sample shows that the as-grown phosphorus doping is indeed very uniform except for some phosphorus loss very close to the surface. Annealing following a silicon

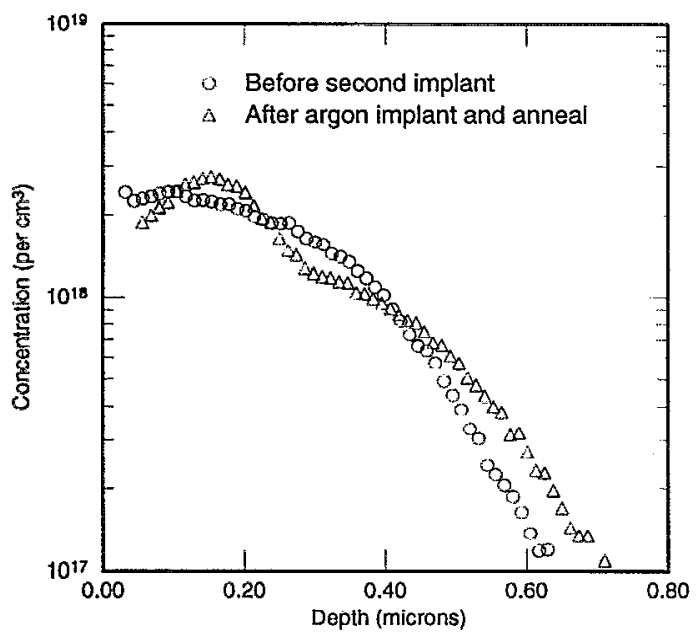

FIG. 2. Transient enhanced diffusion of implanted and annealed phosphorus following a second implantation with argon at $150 \mathrm{keV}$ and dose $7 \times 10^{13} \mathrm{~cm}^{-2}$ and annealing at $800^{\circ} \mathrm{C}$ for $30 \mathrm{~min}$.

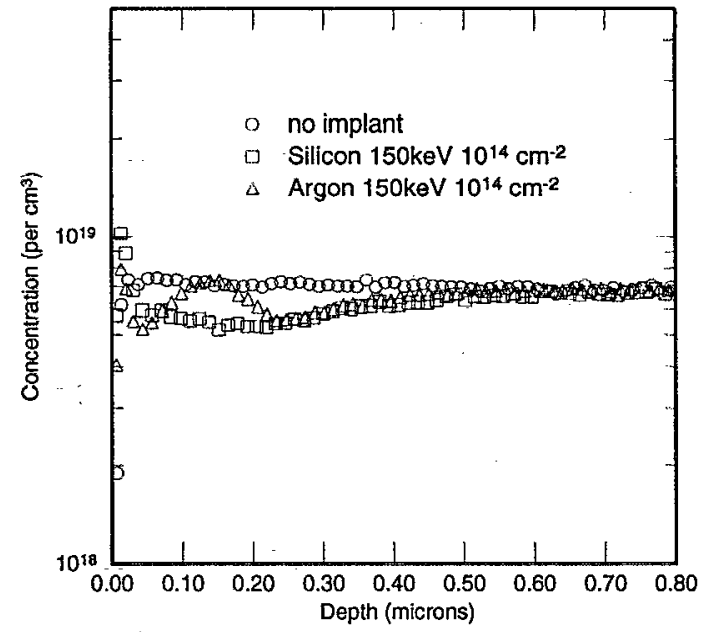

FIG. 3. Transient enhanced diffusion of a uniform phosphorus background following implantation with silicon or argon at $150 \mathrm{keV}$ and dose $7 \times 10^{13} \mathrm{~cm}^{-2}$ and annealing at $800^{\circ} \mathrm{C}$ for $30 \mathrm{~min}$.

implant shows diffusion away from the entire implanted region to the surface. Annealing following an argon implant shows some similarities to Fig. 2, but the phosphorus has not accumulated around $0.15 \mu \mathrm{m}$, but just remained constant. In both cases, implantation damage has driven the phosphorus profile away from its initial uniform state.

The experiments presented here provide further support for a dopant-defect pair model for diffusion. ${ }^{14}$ Since the local pair concentration is proportional to the product of the defect and dopant concentrations, a pair flux can arise from gradients in either the dopant or the defect profile. Since phosphorus is believed to diffuse primarily through interaction with silicon interstitials, ${ }^{15,16}$ interstitial concentration gradients will cause phosphorus diffusion even in regions where the phosphorus concentration is initially uniform. The differences between silicon and argon damage are consistent with this view and show the important role of bulk defect recombination.

Calculation of ion-implantation damage production has shown that there is a small separation between the interstitial and vacancy components of the Frenkel pair distribution. ${ }^{3,17}$ We can consider two limiting cases for the evolution of the defect distributions during annealing. If there is a significant barrier to interstitial-vacancy recombination, excesses of both interstitials and vacancies will remain until diffusion transports them to the surface or into the bulk. Alternatively, if bulk recombination is rapid, the separation between the defect distributions will result in a region of vacancy excess near the surface and of interstitial excess beyond the peak of the implanted profile. Direct $\mathrm{x}$-ray measurements of lattice strain after annealing of high dose silicon implants for $30 \mathrm{~min}$ at $700{ }^{\circ} \mathrm{C}^{18}$ have provided qualitative support for this second view. Those experiments required large doses to yield strain large enough to measure, and a short anneal to restore the lattice crystallinity to allow the $x$-ray technique to be used. The lower doses used here will result in lower point defect con- 
centrations, but their initial spatial distribution should be similar.

The argon damage experiments show strong diffusion away from the region of interstitial excess and no diffusion or dopant pileup toward the region of vacancy excess. This result is consistent with the view that bulk recombination occurs rapidly to form local regions of interstitial and vacancy excess, followed by phosphorus diffusion driven by gradients in the interstitial concentration profile. For the silicon damage case, the addition of one extra interstitial per incident ion dominates the net defect distributions during annealing and leads to an interstitial excess over almost the entire profile, since there are no corresponding vacancies able to remove them. The silicon surface can also play a role as source or sink of point defects, disturbing the defect gradients close to the surface. The diffusion of phosphorus to the surface from the near-surface regions in Figs. 2 and 3 may be attributable to this.

A similar experiment using wafers uniformly doped with antimony, which diffuses via a vacancy mechanism, did not show any measurable change in the antimony distribution following argon implantation and annealing. However, the much smaller intrinsic diffusivity of antimony makes the effect harder to detect. It is interesting to note that Fig. 2 of Pichler et al. ${ }^{14}$ does show diffusion away from the region of vacancy excess. That experiment used high temperature implantation of boron into a wafer with a uniform antimony background, allowing a much greater implantation dose without amorphizing the wafer because the damage was continually being annealed. It supports a vacancy pairing model for antimony diffusion and shows the existence of a residual vacancy excess in the surface region under these conditions even for the case of a pairs +1 implant.

SIMS analysis for part of this work was provided by AT\&T Bell Laboratories.

${ }^{1}$ N. E. B. Cowern, D. J. Godfrcy, and D. E. Sykes, Appl. Phys. Lett. 49, 1711 (1986).

${ }^{2}$ S. Solmi, F. Cembali, R. Fabbri, M. Servidori, and R. Canteri, Appl. Phys. A 48, 255 (1989).

${ }^{3}$ M. D. Giles, J. Electrochem. Soc. 138, 1160 (1991).

${ }^{4}$ M. Servidori, S. Solmi, P. Zaumseil, U. Winter, and M. Anderle, J. Appl. Phys. 65, 98 (1989).

${ }^{5}$ Y. Kim, H. Z. Massoud, and R. B. Fair, J. Electron. Mater. 18, 143 (1989).

${ }^{6}$ M. D. Giles, Appl. Phys. Lett. 58, 2399 (1991).

${ }^{7}$ B. L. Crowder and F. F. Morehead, Appl. Phys. Lett. 14, 313 (1969).

${ }^{8}$ P. Baruch, C. Constantin, J. C. Pfister, and R. Saintesprit, Faraday Soc. Discuss. 31, 76 (1961).

${ }^{9}$ W. Akutagawa, H. L. Dunlap, R. Hart, and O. J. Marsh, J. Appl. Phys. 50, 777 (1979).

${ }^{10}$ Y. Morikawa, K. Yamamoto, and K. Nagami, Appl. Phys. Lett. 36, 997 (1980).

${ }^{1 i}$ S. Loualiche, C. Lucas, P. Baruch, J. P. Gailliard, and J. C. Pfister, Phys. Status Solidi A 69, 663 (1982).

${ }^{12} \mathrm{~S} . \mathrm{M}$. Hu, in VLSI Science and Technology, edited by W. M. Bullis and S. Broydo, (The Electrochenical Society, Pennington, NJ, 1985), Vol. 85-5, p. 465.

${ }^{13}$ G. B. Bronner and J. D. Plummer, Appl. Phys. Lett. 46, 510 (1985).

${ }^{14}$ P. Pichler, R. Schork, T. Klauser, and H. Ryssel, Appl. Phys. Lett. 60, 953 (1992).

${ }^{15}$ P. Fahey, G. Barbuscia, M. Moslehi, and R. W. Dutton, Appl. Phys. Lett. 46, 784 (1985).

${ }^{16}$ P. M. Fahey, P. B. Griffin, and J. D. Plumrner, Rev. Mod. Phys. 61,289 (1989).

${ }^{17}$ L. A. Christel, J. F. Gibbons, and S. Mylroie, J. Appl. Phys. 51, 6176 (1980),

${ }^{18}$ M. Servidori, P. Zaumseil, U. Winter, F. Cembali, and A. M. Mazzone, Nucl. Instrum. Methods B 22, 497 (1987). 